\title{
Pratiques
}

Linguistique, littérature, didactique

\section{Les gestes professionnels dans le modèle de l'agir enseignant : les atouts d'un concept pour la recherche et la formation en didactique du français}

Professional gestures in the model of teaching action: the advantages of a concept for research and training in French didactics

\section{Catherine Dupuy et Yves Soulé}

\section{OpenEdition \\ Journals}

Édition électronique

URL : https://journals.openedition.org/pratiques/10099

DOI : 10.4000/pratiques.10099

ISSN : 2425-2042

Éditeur

Centre de recherche sur les médiations (CREM)

Référence électronique

Catherine Dupuy et Yves Soulé, « Les gestes professionnels dans le modèle de l'agir enseignant : les atouts d'un concept pour la recherche et la formation en didactique du français », Pratiques [En ligne], 189-190 | 2021, mis en ligne le 09 juillet 2021, consulté le 23 juillet 2021. URL : http://

journals.openedition.org/pratiques/10099; DOI : https://doi.org/10.4000/pratiques.10099

Ce document a été généré automatiquement le 23 juillet 2021.

(C) Tous droits réservés 


\section{Les gestes professionnels dans le modèle de l'agir enseignant : les atouts d'un concept pour la recherche et la formation en didactique du français}

Professional gestures in the model of teaching action: the advantages of a concept for research and training in French didactics

\section{Catherine Dupuy et Yves Soulé}

Dès le début des années 2000, nourrie des avancées scientifiques sur l'agir et l'analyse du travail enseignant, la question de la professionnalisation des enseignants s'impose comme un enjeu décisif pour la recherche et pour la formation. Elle conduit une équipe de chercheurs ${ }^{1}$ à élaborer un modèle qui rende compte des caractéristiques favorisant la compréhension, l'exercice et la transformation du métier. Ce modèle, appelé modèle de l'agir enseignant ou modèle $d u$ multi agenda, connait depuis ses premières formulations (Bucheton, Brunet \& Liria, 2005 ; Bucheton, 2009, 2019) un succès à la mesure des applications qu'il autorise mais aussi des controverses qu'il suscite avec des travaux en didactique (Schneuwly, 2008; Sensevy, 2010) ou en analyse psychologique du travail (Clot et Fernandez, 2005). La plupart des collègues en didactique de français qui ont participé à son élaboration ou à sa mise en discussion ont multiplié les recherches pour éprouver sa fiabilité (Bernié \& Goigoux, 2005 ; Bucheton \& Dezutter, 2008 ; Chabanne, Dupuy et al., 2008; Dufays, 2005, 2019) et pointer les apports de la notion de geste mobilisant « une très grande diversité d'approches, d'arrière-plans théoriques, de méthodologies, d'objets... » (Bernié, 2008, p. 237).

2 Le présent article interroge le devenir de ce modèle depuis vingt ans. Une première partie revient sur sa genèse. Une deuxième partie problématise le recours au concept de geste et à ses déclinaisons: gestes de métier, gestes professionnels, gestes langagiers, gestes didactiques... Le terme, ses fondements théoriques, la méthodologie 
d'analyse qui valide son emploi et ses possibles typologies, mais surtout les liens qu'il entretient avec les autres notions du modèle - logiques profondes, préoccupations, postures -, sont-ils suffisamment opérationnels pour en faire un levier de professionnalisation? Que nous disent les travaux qui font de l'analyse par gestes un outil de description et d'interprétation de l'agir enseignant. Jusqu'à quel point un regard, un pointage de la main, un déplacement dans la classe, accompagnés ou non de mots, traduisent une représentation de la situation vécue, une ou plusieurs préoccupations, un changement de posture, une décision? Ces constituants de l'intervention pédagogique et didactique sont-ils pareillement des gestes?

3 En proposant des exemples concernant l'enseignement de la lecture et de la littérature à l'école primaire, une troisième partie évalue le rendement du modèle et du concept pour la didactique du français.

\section{Genèse d'une recherche pour analyser et comprendre le métier enseignant}

4 L'intention première de l'équipe n'était pas de produire des concepts mais des connaissances et des outils d'analyse pour la formation initiale des enseignants en considérant «l'ensemble des activités déployées par les maitres, directement ou indirectement, afin qu'au travers de situations formelles (dédiées à l'apprentissage, mises en place explicitement à cette fin), des élèves effectuent des tâches qui leur permettent de s'emparer de contenus spécifiques » (Crahay \& Dutrevis, 2010, p. 110).

5 Pour rendre compte de cet « ensemble », ont été mobilisés au cours des séminaires et des premiers travaux différents cadres théoriques et méthodologiques permettant d'étudier les pratiques de classe effectives. Il s'agissait notamment de confronter les didactiques disciplinaires - celle du «français » mais aussi des mathématiques - aux théories de l'activité (Theureau, 2004 ; Pastré, Mayen \& Vergnaud, 2006) en faisant du discours des acteurs le matériau privilégié d'une intercompréhension.

\section{Le multi agenda de l'enseignant}

6 Il est important de rappeler le raisonnement qui sous-tend le modèle du multi agenda tel que formalisé par l'équipe du LIRDEF (Bucheton \& Soulé, 2009) et trop souvent réduit dans les travaux qui le citent à la seule notion de « geste ». Lorsqu'un enseignant conçoit, conduit, commente une séance d'enseignement/apprentissage, il le fait à partir de la situation vécue, de ses connaissances, de l'expérience acquise, de ses rapports aux savoirs à enseigner, aux instructions officielles, aux élèves, au langage, aux émotions... Ces différents rapports à ont été définis comme logiques profondes au sens où elles sont rarement conscientisées et difficiles à verbaliser, ou comme logiques "d'arrière-plan" au sens où elles ne participent pas directement à l'élaboration, au déroulement ou au commentaire des séances mais conditionnent chacune de ces phases.

7 Ces logiques structurent en effet la représentation que l'enseignant se fait de la situation et des préoccupations qu'elle induit. Cinq préoccupations récurrentes ont été repérées : l'atmosphère qu'il juge indispensable au déroulement de la séance, le pilotage de l'espace, du temps, du rythme, des tâches, des interactions, les procédures d'étayage mobilisées 
pour aider les élèves, le tissage, c'est-à-dire l'explicitation des liens entre les phases de la situation, avec d'autres situations antérieures ou postérieures, enfin et surtout nous y reviendrons - la transmission effective des contenus d'enseignement.

Ces préoccupations déterminent un choix de postures - manières d'être, de penser et d'agir (Bucheton et al., 2008, 2019) - comprises entre le contrôle, l'accompagnement, le lâcher-prise et la posture dite du magicien, produisant le savoir sans l'expliciter

Dernière étape de la modélisation, postures et préoccupations s'actualisent dans des gestes. Autrement dit, avant même que ne soit instruit son procès en conceptualisation, le mot a d'abord été choisi, au plus près de son étymologie latine (gerere = porter, produire, mener à bien, se charger de, administrer) pour désigner ce que l'enseignant fait, décide de faire, doit faire, dit de faire. Préoccupations et postures sont donc enchâssées et fonctionnent de manière systémique. Les gestes qui en rendent compte sont dès lors multiples dans leur enchainement et "épais » dans leurs significations. D'où la notion de multi agenda configuré dans le schéma ci- dessous :

Schéma du multiagenda. Source : Bucheton \& Soulé, 2009

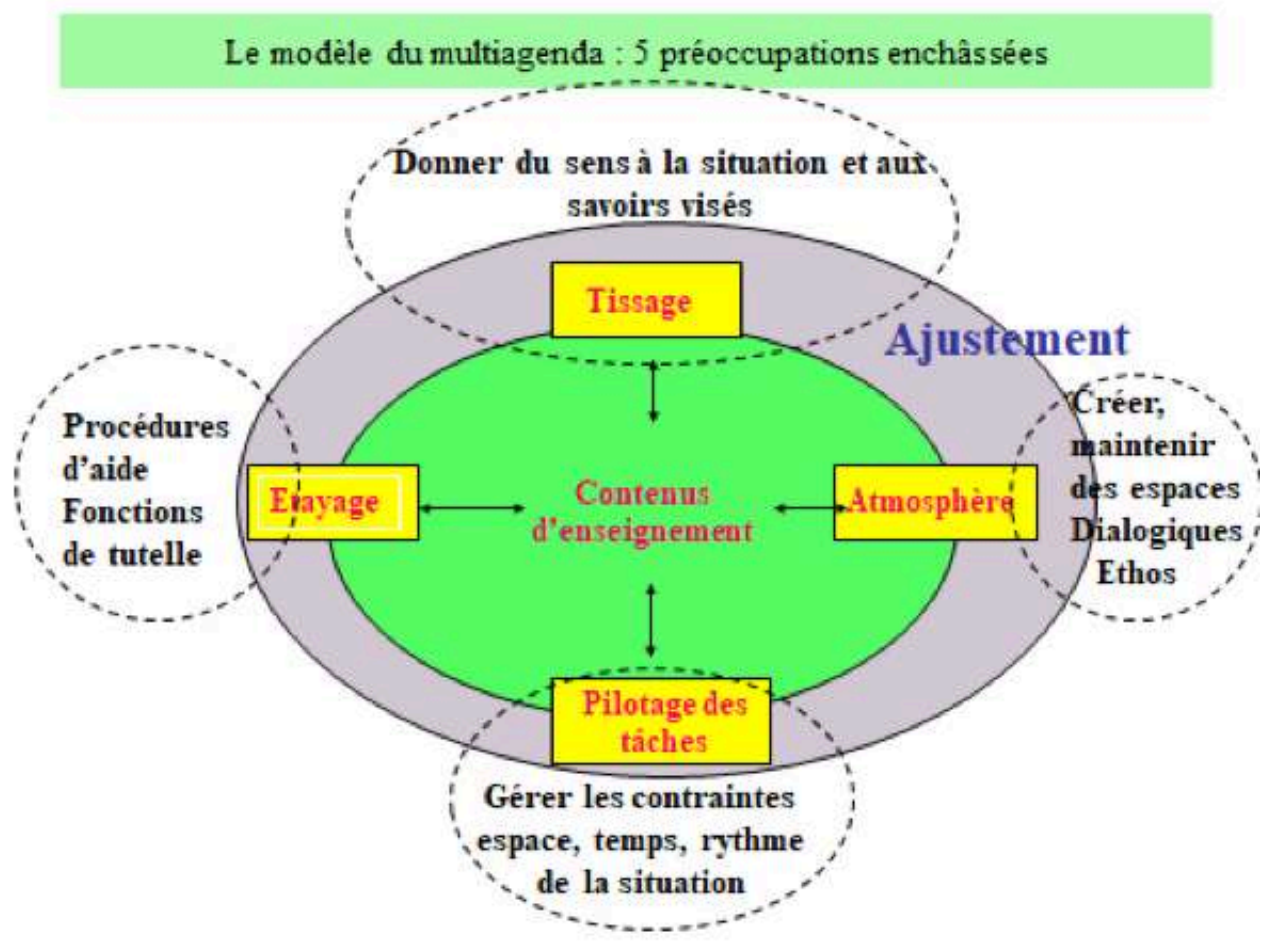

10 Amené à traiter tout à la fois les contenus, le temps, les aides, le comportement des élèves, les discours, l'enseignant ajuste son action en effectuant des gestes qui, en première analyse, sont autant de ressources plus ou moins consciemment orientées dans et pour l'action.

\section{Un souci de conceptualisation?}

11 L'identification des gestes - Qu'est-ce qui fait geste ? Et pourquoi ce terme ? - part donc de la volonté de documenter l'agir enseignant en rassemblant et en catégorisant des théories contributives, des pratiques, des discours, des concepts adjacents. Dans cette perspective, le travail de validation conceptuelle était indispensable mais, dans une 
certaine mesure, second. En effet, si un concept se définit comme « une construction rendant compte de caractéristiques communes à un ensemble d'objets, de faits ou de phénomènes » (Reuter, 2013, p. 35), le recours au mot geste s'inscrivait d'abord comme élément d'une "construction » qui part des problèmes que pose la caractérisation du travail de l'enseignant et qui, en retour, oblige à problématiser comme instrument de connaissance chacun des termes retenus: geste, mais aussi posture, préoccupation, représentation, logique profonde.

D'autre part, comme le rappelle Dumez, « les concepts ne sont définis au départ que sur un mode provisoire, cette définition devant constituer l'orientation du travail, ils sont ensuite redéfinis au cours du processus de recherche. Le processus de recherche étant justement ce travail de redéfinition» (Dumez, 2011, p. 78). La question de savoir si, dans ce "processus de recherche", l'analyse par gestes opère le "travail de redéfinition" attendu, permet en outre de relativiser l'«indétermination conceptuelle » de la notion (Cizeron, 2010). En revendiquant l'analyse psychologique du travail (Clot, 1999) et la part phénoménologique de l'action humaine (Jorro, 1998, p. 10), le concept de geste, tel que nous l'entendons, désigne les composantes significatives de l'agir enseignant dans toute son " épaisseur » (Bucheton, 2009, p. 54).

L'assise théorique ainsi définie, ces "composantes» ne se limitent pas aux actions d'expertise - le bon geste -, elles intègrent aussi bien les dimensions visibles de l'agir, corporelles et langagières, que les dimensions cognitives et abstraites qui sous-tendent « la pensée des enseignants pendant l'interaction » comme autant de «choix d'actions parmi une panoplie d'alternatives » (Wanlin \& Crahay, 2012, p. 17).

14 L'avancée conceptuelle légitime de fait la multiplicité des travaux et des motivations concernant l'engouement pour le mot geste, comme le montre J.-P. Bernié (2008, p. 238) :

Le surgissement d'intérêt pour la question est général, mais dans une dispersion qui reflète la très brève histoire du champ : certains y sont entrés à partir du souci de faire évoluer les didactiques vers une meilleure prise en compte du réel de la classe, d'autres à partir du souci de mieux définir les objets d'enseignement; certains à partir de recherches en psychologie cognitive, d'autres à partir de points de vue sociologiques sur les raisons des effets différenciateurs des pratiques d'enseignement, d'autres enfin à partir d'un cheminement les conduisant à privilégier la description de la professionnalité.

15 Mais plutôt que parler de "dispersion", l'approche par gestes permet au contraire de combiner analyses de pratiques, analyses de l'activité, enjeux didactiques et réflexivité (Schön, 1983). Quel que soit le « cheminement » théorique, la « prise en compte du réel de la classe", l'attention portée aux "objets d'enseignement" ou aux "effets différenciateurs » sont nécessaires, à «la description de la professionnalité ». Le modèle du multi agenda et le recours au mot "geste " traduisent cette tentative d'intégration au bénéfice d'une meilleure évaluation de l'activité enseignante, comme en témoigne G. Sensevy : « la notion de geste est contemporaine d'une reconnaissance : la reconnaissance dans le travail du professeur d'une richesse et d'une complexité qu'il faut apprendre à décrire» $(2005$, p. 4). Et ce n'est pas parce que les enseignants n'utilisent pas le mot (Cizeron, 2010, p. 9) que ce qu'ils disent de leur activité ne peut pas être défini comme tel.

16 Les emplois du mot geste ont ainsi généré une dynamique de validation conceptuelle répondant aux trois caractéristiques du triangle d'Ogden et Richards (1923) que reprend H. Dumez dans Qu'est-ce qu'un concept ? (2011) : la dénomination (le nom donné), 
la compréhension (la définition) et l'extension (les cas empiriques auxquels s'applique le concept), ceux-ci entretenant des interactions dynamiques. En donnant comme définition au mot geste la «manifestation motrice et/ou verbale de l'activité » (Chabanne \& Dezutter, 2011, p. 11-12), on part d'une compréhension et d'une extension minimales efficaces : le geste étant « la forme visible, aisément observable, éventuellement reproductible» de cette activité, l'analyse par gestes permet bien d'étudier le travail enseignant.

En se référant enfin aux huit critères de Gerring (1999) que mobilise également H. Dumez (2011), les mots du modèle, "geste», mais aussi «préoccupation » ou " posture » - répondent à quatre d'entre eux, en ce qu'ils ont acquis au fil des travaux dans la communauté scientifique une "familiarité » ("manifestation motrice et/ou verbale»), une "résonance» (efficacité métaphorique du mot "geste»), une " cohérence » et une " utilité pour le champ sémantique » en particulier en formation. En revanche plus discutables, et par là-même intéressants à approfondir, sont les critères de "parcimonie " (les définitions du mot geste seraient trop limitées, trop larges ou trop métaphoriques), de « différenciation » (par rapport à d'autres concepts voisins : "schème", "technique »), de "profondeur " (capacité à "regrouper " des caractéristiques précises), d'« utilité théorique » compte tenu par exemple des liens que le concept de geste entretient dans le modèle avec celui de préoccupation, lequel est aussi présent dans la théorie du cours d'action (Theureau, 2004). La deuxième partie de cet article poursuit dans ce sens le travail de problématisation.

\section{Le recours à l'analyse par gestes}

\section{Distinguer gestes de métier et gestes professionnels}

Existe-t-il une logique dans les agencements sémantiques qui ont ponctué, dès les premiers travaux de l'équipe de Recherche Technologique, la montée en puissance du concept de geste et de ses déclinaisons ? Pour répondre à cette question, il faut d'abord revenir à la distinction entre geste de métier et geste professionnel

Pour A. Jorro, au plus près de la réalité physique du métier, le geste est d'abord : « un mouvement du corps, adressé, porteur de valeurs et inscrit dans une situation, irrigué par la biographie et l'expérience du sujet » (Jorro, 2005). La conception de l'agir humain est d'ordre symbolique, expérientielle et située. De fait, un geste est « une action de communication inscrite dans une culture partagée, même à minima. Il prend son sens dans et par le contexte scolaire » (Bucheton \& Soulé, 2009, p. 32).

Le concept, soulignant l'importance de la corporéité de l'activité, invite également à réfléchir à la dimension multimodale et pluridimensionnelle des interactions en contexte scolaire : le « corps parlant » de l'enseignant (Jorro, 2005, op. cit.) se décline en gestes physiques et gestes langagiers, fortement codés, qui agissent aussi bien au plan socioaffectif que cognitif. Ils sont transversaux et «au-delà de leur caractéristique technique, portent et traduisent avant tout la symbolique des principales actions, actes, gestes ou micro gestes techniques d'un métier (dicter, questionner, corriger au tableau, se déplacer, noter, évaluer, etc.) » (Alin, 2010, p. 54).

21 Deux autres points sont à signaler dans les écrits d'A. Jorro. Le premier concerne la notion de kairos, c'est-à-dire la capacité de l'enseignant à saisir le "moment opportun " pour assurer la transmission optimale du savoir et l'efficacité de l'apprentissage dans le 
processus d'adressage à autrui. Le second tient à la distinction trop souvent occultée ou différemment comprise entre gestes de métier et gestes professionnels. "Objet d'une transmission technique et symbolique, le geste du métier véhicule les codes propres au métier » (Jorro, 2005, p. 8) alors que les gestes professionnels « dépendent de processus d'ajustement, d'agencement, de régulation " (ibid.). De nature plus réflexive, ils intègrent les gestes de métier et comprennent les dimensions singulières d'une cognition et d'une émotivité toujours situées.

Si un geste de métier " peut être repéré et faire l'objet de tentatives de modélisation parce qu'il renvoie à un répertoire de pratiques répondant à un certain degré de codification et de stabilité » (Chabanne \& Dezutter, 2011, pp.11-12), il comprend toujours «une part indispensable d'improvisation et d'ajustement à l'instant de l'action " (ibid.) où se lit la professionnalité du geste. Par conséquent, on peut (a) décider d'insister sur sa dimension sociale, qui renvoie aux normes, aux pratiques héritées de la culture scolaire, aux injonctions, aux rituels didactiques, (b) privilégier les « arts de faire et de dire » qui donnent à voir chez l'enseignant l'actualisation de ses préoccupations et orientent sa conduite de classe, (c) admettre, sans affaiblir la distinction entre les deux concepts, que sous l'effet conjugué des normes, des recherches et des pratiques observées, un geste professionnel, autrement dit « un art de faire et de dire », peut devenir un geste de métier. La dictée à l'adulte en maternelle (qui permet aux élèves non scripteurs de produire un texte écrit sous leurs yeux par l'enseignant expert) formalisée par M. Brigaudiot (1995) ou le travail de compréhension des textes didactisé par S. Cèbe et R. Goigoux dans Lector \& lectrix (2009) sont de bons exemples : ils sont issus d'expérimentations, accèdent au statut de méthode d'enseignement / apprentissage reconnue, avant d'être à leur tour re-professionnalisés dans des pratiques ajustées.

Un dernier élément à souligner concerne la dimension langagière de l'activité et du discours sur l'activité. Le postulat de départ est qu'« on ne peut rendre compte de la coactivité maitre-élèves sans analyser son vecteur principal : les langages partagés. Ils en sont les médiateurs et la trace. Ils sont les instruments principaux de la construction des savoirs » (Bucheton, 2005, p. 19). La position est ici proche de celle de C. Alin pour qui « comprendre et expliquer cette dimension langagière et pragmatique (...) est l'un des apports majeurs d'une grammaire analytique des pratiques » (Alin, 2010, p. 51). Elle consiste à étudier systématiquement le fonctionnement des formes d'interpellation, de feed-back, de reformulation notamment à l'adresse des élèves en difficulté (GarciaDebanc \& Volteau, 2007). Les gestes de l'enseignant interagissent avec les gestes d'étude des élèves pour résoudre des classes de problèmes dans des contextes didactiques précis, si bien qu'ils "peuvent être définis comme les micro-activités langagières et corporelles que le maitre et les élèves effectuent tant dans le cadre de la classe qu'en préparation ou en prolongement de celle-ci »(Marlair \& Dufays, 2008, p. 61).

\section{Démultiplier les types de gestes : une granularité nécessaire}

Depuis que le modèle intéresse la communauté des chercheurs et des formateurs, le répertoire des gestes identifiés n'a cessé d'augmenter : le générique geste et le doublet geste de métier / geste professionnel ont été déclinés en geste didactique, geste langagier, geste témoin, geste lexique. Une typologie aussi brouillonne semble fragiliser l'édifice conceptuel - «Tout est geste et le geste est tout» (Bernié, 2008, p. 239). Sauf à 
considérer qu'un marquage plus fin contribue à la vitalité et à l'opérationnalité du concept.

Dans une séance consacrée à un apprentissage lexical, définir un terme pour répondre à la sollicitation d'un élève constitue un geste professionnel amenant l'enseignant à ajuster ses possibles : proposer à la classe de trouver la définition? revenir au texte pour saisir le sens du mot? s'aider d'un dictionnaire? différer la définition?). Mais ce geste est en même temps langagier (mise en mots particulière de la réponse et choix d'une posture adaptée ; "Attendez, avant de répondre à votre camarade, j'aimerais qu'on continue de lire un peu le texte...»), il répond à une intention didactique (concernant par exemple la découverte d'un mécanisme d'affixation) et peut être qualifié de témoin au sens où précisément il permet d'identifier et de questionner une conduite d'explication à un moment donné d'une séance de lecture littéraire. Autrement dit, en faire à la fois un geste professionnel, langagier, didactique, témoin, fédère un ensemble de points de vue nécessaires pour saisir la complexité de l'agir et structure les différentes strates typologiques

La recherche menée par Dupuy \& Genre (2020) s'inscrit dans cette logique : le « geste lexique » représente une sous-catégorie des gestes et préoccupations professionnelles d'étayage dans les interactions portant sur le vocabulaire des textes. Il est d'autant plus intéressant à isoler qu'il constitue une pratique récurrente (savoir expliquer un mot aux élèves est un geste de métier), mais cette tâche « doxique » (Dupuy, 2009) dépend d'un savoir-faire finalement peu didactisé, zone aveugle de l'enseignement de la lecture littéraire. En effet, lors des interactions orales, la compréhension d'un mot se fait soit en amont du travail sur le texte, soit dans le pas à pas de la rencontre avec un terme jugé "difficile", soit à partir des mouvements approximatifs d'approche du sens et d'hypothèses d'une définition mise en attente. Le choix engage donc une professionnalité singulière qui autorise le grain de catégorisation retenu.

\section{Pour un inventaire stimulant des critiques et limites concernant l'usage du concept et du modèle}

27 L'utilisation de l'analyse par gestes pour la recherche et/ou la formation dans différentes didactiques (Bucheton, Bronner, Broussal \& Larguier, 2006; Bucheton \& Dezutter, 2008; Chabanne \& Dezutter, 2011; Bonnard \& Detalle, 2014; Lhoste, Champagne \& Bulf, 2016) montre que le succès du concept de geste et du modèle est à la mesure des critiques qui lui sont adressées et des limites éprouvées lors de son exploitation. On peut en identifier trois.

- L'ancrage théorique

28 L'adhésion au modèle et au concept dépend de motivations différentes (Bernié, 2008, p. 3). Elle conduit à combiner ou non des champs scientifiques a priori distants mais nécessaires si l'on veut comprendre l'activité enseignante. Plusieurs problèmes se posent alors. Le premier consiste à refuser tout écart théorique donnant au mot "geste " une définition extensive : seule la dimension physique, corporelle - " action motrice intentionnelle » (Cizeron, 2010, p. 3) - serait à prendre en compte, seul serait qualifié de geste le geste expert. Le deuxième tient à la complémentarité entre les champs qui doit être clairement établie. Un exemple montre bien l'intérêt d'options théoriques contributoires : R. Goigoux a recours à la notion de schème comme «forme organisée et stabilisée de l'activité d'enseignement pour une certaine variété de 
situations appartenant à une même classe " $(2005$, p. 7$)$. Il définit ainsi le geste professionnel en tant qu'il combine des structures invariantes propres à l'exercice du métier. Le recours aux schèmes permet également à J. L. Dufays de montrer «qu'il existe bien un certain nombre de gestes et d'attitudes spécifiques tant à l'enseignement qu'à l'appropriation du fait littéraire »(2005, p. 12-14). Il n'y a donc pas d'incompatibilité entre les cadres cognitif, didactique et ergonomique. Le troisième écueil est celui d'une désolidarisation des supports théoriques : c'est le cas lorsque le concept de geste est coupé du modèle dans lequel il a été construit ou qu'un énoncé est qualifié de "geste de... » sans aucune référence aux critères de l'analyse linguistique et interactionnelle.

- La méthodologie d'identification et d'interprétation des gestes

Nombre des remarques sur le concept de geste procèdent d'une lecture partielle (recontextualisation insuffisante) du modèle de l'agir professionnel. Des raccourcis sont trop souvent faits assimilant préoccupations et gestes. On oublie non seulement le principe d'enchâssement des préoccupations mais aussi le caractère "épais » des gestes.

30 Un même geste peut en effet répondre à un ordre de préoccupations particulier. Au terme d'une leçon de langue, un enseignant annonce : « Nous verrons demain quel nom donner à cette notion, pour l'instant retenez que...». L'ajustement temporel s'impose (préoccupation première: le cours s'achève), mais, dans le mouvement de l'énoncé, le tissage (deuxième préoccupation : demande de mémorisation) dit la mise en suspens de la transmission du savoir (troisième préoccupation: progresser dans la compréhension de la notion). À moins que tout cela soit un choix didactique délibéré (véritable préoccupation qui ne serait pas liée à un défaut de pilotage du temps de la séance), le scénario didactique envisagé étant bien celui d'une co-construction progressive de la notion.

31 De la même manière, un geste peut renvoyer à une combinaison de préoccupations. L'énoncé suivant, «Je vous laisse deux minutes pour relire le texte ", accompagné d'un pointage de la main sur le texte, peut signifier à la fois le souci de maintenir les élèves dans l'activité (préoccupation relative à l'atmosphère et au pilotage : assurer le tempo de la lecture à un moment où la classe bute sur un passage difficile); de progresser dans la lecture du texte (préoccupation relative à l'objet d'enseignement : lever un obstacle de compréhension), d'aider les élèves (préoccupation d'étayage : la relecture comme procédure de traitement des informations).

32 Par conséquent, la difficulté est ici d'ordre méthodologique. L'entreprise qui consiste à isoler un geste est toujours problématique, car l'improvisation et/ou l'ajustement des gestes dépendent de l'évolution de la situation et des modifications parfois minimes du geste de métier (présenter une leçon grammaticale, lire un texte littéraire). L'identification et le comptage des gestes s'avérant difficile, tout traitement statistique est discutable si les gestes ne sont pas compris dans leur "dynamique " signifiante (Bucheton \& Soulé, 2009, p. 45 ; Jaubert \& Rebière, 2019).

- La force centrifuge du modèle

En considérant que l'adjectif « professionnel » dans l'expression " geste professionnel » signifie "du métier", autrement dit "des gestes dont le système constitue une structure spécifique de ce métier » ( 2005, p. 4), puis en introduisant la notion de « geste d'enseignement» $(2010$, p. 4), c'est-à-dire geste du savoir enseigné, G. Sensevy 
formule une autre critique adressée au concept. La question est de savoir s'il ne conduit pas à une "dichotomie » contre-productive entre " gestion » et « didactique » (2010, p. 2). Ce problème concerne également l'exploitation du modèle. Les étudiants qui dans leurs travaux ${ }^{2}$ exploitent le modèle comme cadre théorique et/ou outil d'analyse de leurs données ont tendance à privilégier les préoccupations périphériques (atmosphère, pilotage, étayage, tissage), à les traiter dans leur dimension pédagogique, au détriment de la préoccupation, placée au centre du schéma : le savoir à enseigner. Autrement dit, la force centrifuge du schéma entrainerait "l'oubli des savoirs» (Sensevy, 2010, p. 3). Non seulement ils ne les voient pas comme la "visée organisatrice " de l'activité (Bucheton, 2008) mais ils oublient aussi la part didactique des préoccupations. Une mise au point s'impose alors pour repenser l'enchâssement des cinq préoccupations, éviter toute «dichotomie » et recourir au concept de geste pour « cristalliser la substance même du savoir dans les interactions et les transactions entre élèves et professeur » (Sensevy, 2005, p. 5).

\section{Un concept et un modèle rentables pour la didactique du français}

Cette dernière partie envisage l'efficacité de l'analyse par gestes pour l'enseignement de la lecture et de la littérature. Ayant œuvré, depuis l'émergence du champ, plutôt dans une logique d'intervention sur les pratiques (conception, évaluation), la didactique du français s'est progressivement intéressée à l'activité réelle de la classe et au partage possible avec d'autres didactiques pareillement impliquées dans les questions de formation. Trois raisons peuvent expliquer cet ancrage : (a) l'élaboration conceptuelle nécessaire pour inscrire dans la durée la légitimité de la discipline, (b) la prise en compte de l'analyse de l'activité comme paradigme de recherche et de formation, (c) le fait que des fondements théoriques du modèle des gestes professionnels entraient en adéquation avec les concepts construits par les didacticiens du français.

On retient l'hypothèse, formulée par J. P. Bernié, « qu'une extension de la recherche en didactique vers une discipline de formation [est] à construire» (2009, p. 245) et, dans le même temps, que l'agir enseignant participe amplement « au processus de construction permanent d'une discipline en train de se faire » (Bulea Bronckart, Ronveaux \& Védrines, 2019, p. 243).

Dans cette perspective, la fonction du modèle est essentiellement euristique, particulièrement pour la formation initiale. L'économie du concept - ses usages et ses valeurs - profite autant de l'entreprise de modélisation dans laquelle il s'inscrit que des limites que nous avons relevées. L'analyse par gestes permet de comparer des pratiques pour qualifier des situations d'enseignement, dégager des régularités, valoriser des " arts de faire » singuliers (De Certeau, 1990, p. 102). Elle met des mots sur le réel de l'activité et sur la pensée de l'activité. Elle aide au développement de compétences d'identification des composantes de l'activité : qualifier de geste un mouvement, un énoncé, une pensée, élaborer un travail interprétatif le rattachant à des préoccupations, à des postures, à des logiques profondes autorise un exercice réflexif qui donne à voir les compromis opératoires, les dilemmes auxquels l'enseignant doit répondre. 
Son efficacité tient aussi aux modalités de recueil des données issues des recherches orientées activité. L'introduction du support vidéo et les corpus d'entretiens ont profondément transformé l'ingénierie traditionnelle de l'analyse de pratiques $^{3}$. Les didacticiens du français qui avaient déjà largement intégré l'usage des verbatims, se sont emparés de la vidéographie et de la vidéoformation, associant séances filmées et entretiens. Ils complètent ainsi les moyens d'identification et de compréhension du travail disciplinaire. La didactique de l'oral, comme outil d'enseignement et objet d'apprentissage, bénéficie largement de cet outillage méthodologique qui appréhende la réalité multimodale des interactions verbales, dès lors plus facilement observables et commentables en termes de gestes professionnels langagiers didactiques. En témoignent les travaux sur les reformulations orales (Garcia-Debanc, 2019; Bulea Bronckart, 2010) qui désignent ces phénomènes de reprise comme marqueurs d'expertise (donc comme des gestes professionnels langagiers et didactiques significatifs) et autorisent leur travail en formation. Le concept de geste joue ici un double rôle de révélateur professionnel et de médiateur entre la recherche et la formation, entre didactique et la compréhension de l'agir. Si tout s'organise en fonction des contenus et des choix didactiques, tout ne saurait être didactique: les gestes langagiers de lecture, les gestes lexique, les gestes de pointage d'une notion, de « mise en scène du savoir » (Jorro, 2002), s'accordent aux gestes d'ajustement sans les intégrer totalement.

\section{Donner à voir et à commenter l'activité en «français " pour former}

S'ajoutant aux différents dispositifs d'analyse des pratiques, le modèle et le concept de geste accompagnent la "prise de conscience» du travail enseignant (Bulea \& Bronckart (2010, p. 52). Ils fonctionnent comme un matériau de la production discursive abordant l'agir enseignant (physique et symbolique) sous l'angle du raisonnement professionnel. Dès lors que la didactique s'intéresse à la formation, parler de geste de relecture, de geste d'explicitation, de geste lexique c'est identifier en situation les modalités (et les difficultés) d'application des principes d'action qu'elle édicte et qui sont trop souvent considérés par les enseignants comme "théoriques". L'analyse par gestes recentre ainsi l'attention sur la dimension praxéologique de la didactique et autorise son intégration à la matrice épistémologique de la discipline. Si l'on admet avec E. Nonnon (2017, p. 15; Nonnon, 2019 ) que «la question de la verbalisation et de la réflexivité se pose en formation d'enseignants relativement à chacune de ses dimensions (épistémique, pragmatique, identitaire) et aux interactions entre elles ", cet outil d'analyse multidimensionnelle et multiréférentielle contribue à les documenter.

De fait, le décryptage des gestes éclaire les situations d'enseignement/apprentissage dans toutes les composantes de la discipline. Il permet d'articuler les savoirs disciplinaires (et les problèmes que pose leur transposition didactique) aux savoirs professionnels qui combinent les invariants et les singularités de l'agir. Ils fournissent une grille de lecture au service de la documentation des pratiques, ajustée aux possibles des enseignants et à leur compréhension des prescriptions. La circulation sur les différents niveaux du modèle permet une recherche à dominante clinique compatible avec une expérimentation et des techniques euristiques d'une méthode empirique qualitative à partir d'un terrain d'étude circonscrit par la problématique 
formulée autour d'entretiens, questionnaires mais principalement d'observations de classes dont celle du stagiaire.

\section{La démarche d'analyse, véritable enjeu de l'exploitation du concept}

Dans notre expérience de formateur, la réception du concept est le plus souvent positive : les étudiants déclarent qu'il leur permet d'interroger les pratiques de classe, de requalifier leur expérience d'enseignement, de répondre aux standards de la recherche (problématisation, outil d'analyse des données et production de résultats). Mais sa véritable efficacité euristique réside dans la démarche adoptée pour éviter les écueils relevés dans la deuxième partie: identification des gestes sans référence au modèle dans sa totalité, confusion entre préoccupation et geste, tentation applicationniste et «oubli des savoirs»(supra, p. 8). Les trois exemples que nous donnons ici montrent quelles sont les modalités de cette démarche à partir du support que constituent à minima l'enregistrement d'une séance et l'entretien avec l'acteur. Dans le premier, il s'agit de savoir comment on peut inférer des préoccupations, gestes et postures à partir des descriptions et des commentaires qu'un enseignant propose de son activité. Le second montre comment fonctionne l'analyse par gestes et comment la présentation du modèle peut impacter le développement professionnel d'un enseignant. Le dernier approfondit la démarche d'analyse autorisant l'emploi du mot geste.

- Premier exemple : l'étude d'un entretien d'autoconfrontation dans le cadre d'une recherche sur l'enseignement de la lecture et du lexique en classe de littérature (Dupuy, 2009).

41 Des enseignant.e.s de cycle 3 (CE2, CM1) décrivent leur activité au début d'une séance consacrée au texte de Bernard Friot, Archimémé (Histoires pressées, 2005). Ni le modèle de l'agir ni le concept de geste n'ont été évoqués en amont de l'entretien.

Extrait $1:$ Pierre

Pierre : Bon / là... j'ai beaucoup LE DOIGT LEVE !(rires)

Chercheur: là y a des choses qui se condensent là / vas-y tu peux commenter

Pierre: là trois groupes trois compétences particulières et des techniques différentes qui

doivent normalement faire émerger par /la mise en commun /qui doivent faire une construction collective du sens ...ah donc là /y a le... je suis en position d'animateur / D'ANIMATEUR DE CENTRE CULTUREL// mais je suis aussi en train de mettre en place tout ce qui s'est passé dans les groupes /et là/// c'est là où ça devient en principe /et en principe là je choisis des textes qui comportent une énigme et c'est à ce moment-là qu'on est censé tous ensemble résoudre l'énigme //donc je rappelle qu'un tel a dit ça parce que voilà je le montre /donc/je me déplace /je suis plus dans la position de metteur en scène en train de répliquer à des acteurs /qu'il fallait lire ça comme ci comme ça//là je laisse je dramatise un peu!(rires) je me vois en train de lever le doigt /je savais pas que je le faisais aussi souvent //j'avais une crampe à l'index!

Ce verbatim donne à entendre une conception de l'enseignement de la littérature au cycle 3 qui relève de l'acculturation, de la compréhension de la lecture par résolution d'une " énigme » et partage du sens du texte. En visionnant la séance (reprises de l'adverbe « là ", l'enseignant évoque des préoccupations didactiques (nous sommes ici au centre du modèle) qui, sans être vraiment explicitées et assumées, comme le montrent les modalisations ( normalement »; «en principe»; «on est censé »), se traduisent par des gestes littéralement remarquables ( «j'ai beaucoup LE DOIGT LEVE »; «je me vois en train de lever le doigt /je savais pas que je le faisais aussi souvent //j'avais une 
crampe à l'index !». Ces commentaires rendent compte de la théâtralisation des interactions. L'enseignant, surpris, mesure l'importance de la posture corporelle, langagière et mentale qu'il adopte : d'« animateur de centre culturel», il devient «metteur en scène en train de répliquer à des acteurs ». La médiation du modèle complète et conforte l'analyse de cette scénographie par l'acteur, elle permet d'identifier des gestes professionnels de régulation en lien avec une organisation didactique diversifiée (" trois groupes, trois compétences particulières et des techniques différentes ")qui répond à la volonté de créer une atmosphère propice à la mise en commun.

Extrait 2 : Annick

Chercheur: alors là, qu'est-ce qui se passe?

Annick: alors /si tu veux /au départ /c'est pas de moi /c'est ma collègue elle/ elle insiste BEAUCOUP ECRIVEZ DES MOTS donc/ c'est quelque chose qui me semble indispensable/... mais je sais pas si j'ai mis toute la conviction qu'il fallait/...mais je me suis dit qu'ils aient au moins un truc à dire/ mon but c'est le débat je l'ai dans la tête mais je fais faire quand même le passage à l'écrit comme je l'ai vu faire l'année dernière et je l'ai fait en étant partiellement convaincue...// c'est pour ça que la didactique du français tu vois j'y crois qu'à moitié// mais je pense que le débat littéraire ça permettra au moins d'écouter d'avoir d'autres activités d'interprétation possibles /et en revanche je crois davantage à la trace écrite à la fin où chacun garde quelque chose/sachant que chez moi c'est pas très au point parce ce n'est pas suffisamment structuré et quand on a lu rapidement ce qu'ils avaient écrit à la fin /c'était pas terrible... ben/ c'était pas terrible /c'était pas très construit

L'enseignante évoque le passage par l'écrit dans le cadre d'un débat littéraire. Si elle est convaincue de l'intérêt d'une "trace écrite " à la fin de l'activité (geste de métier stabilisé), faire écrire des mots aux élèves avant le débat est un principe d'action ("c'est quelque chose qui me semble indispensable») qu'elle peine à appliquer. Qualifier de préoccupation son hésitation à piloter le temps d'écriture de part et d'autre du débat aide à dissocier les savoirs d'expérience et les savoirs académiques, à reconsidérer les préconisations didactiques jugées peu convaincantes.

Avec l'acteur dans un accompagnement spécifique comme en formation auprès d'un collectif d'enseignants, l'analyse par gestes invite à une économie de l'attention que privilégie les indices linguistiques et proxémiques. Seuls les allers-retours entre la vidéo et l'autoconfrontation ainsi que l'examen outillé des formulations langagières permettent l'identification et la catégorisation des gestes, préoccupations et autres postures. Ainsi, dans les propos de Pierre: ah donc là /y a le... je suis en position d'animateur /D'ANIMATEUR DE CENTRE CULTUREL// mais je suis aussi en train de mettre en place tout ce qui s'est passé dans les groupes, peut-on noter au fur et mesure de l'indexation des actions observées une concomitance d'actions et de postures (animateur et enseignant) visant la conservation de la maitrise de la classe.

L'enjeu n'est donc pas de montrer une bonne pratique mais de mettre à jour des indicateurs qui définissent au mieux les composantes d'une pratique. Les enseignants confrontés aux traces audiovisuelles de leur activité élaborent un discours pour l'essentiel monogéré qui a pour rôle fonctionnel de leur faire passer «un palier praxéologique » (Chevallard, 2003, p. 2), celui de la prise de conscience de l'expérience de la classe. Le recours au modèle autorise une formalisation langagière intersubjective tenant de la résolution de problème (Vygotski, 1995, p. 329).

- Deuxième exemple: une thèse en cours sur la formation hybride d'enseignant.e.s débutant.e.s enseignante à partir de traces vidéo d'une séance de "français" portant sur les 
invariants du roman policer ${ }^{4}$. Il croise de manière systématique une analyse quantitative et qualitative des gestes observés qui doit encore faire l'objet d'un examen critique pour stabiliser les critères d'attribution de tel ou tel geste avec une ou plusieurs préoccupations. Pour l'heure, il avance les premiers résultats :

Le savoir apparait comme une préoccupation centrale. $50 \%$ des orientations thématiques lui sont consacrées. Un quart porte sur l'étayage. L'atmosphère ainsi que le tissage sont très faiblement thématisés (5\%), de même que le pilotage (16 \%). $70 \%$ des propos thématisent les relations entre la préoccupation du savoir et celle de l'étayage et/ou du tissage ; $20 \%$ les liens entre pilotage et atmosphère. Les $10 \%$ restant évoquent un rapprochement entre pilotage et étayage.

Il montre que l'enseignante contrôle les interactions mais note cependant "une tentative d'orientation vers une posture d'accompagnement et, d'autre part, ce qui apparait comme l'intention de vouloir remettre la construction du savoir entre les mains du collectif ». Après une formation sur le modèle de l'agir enseignant à laquelle participe l'enseignante, il constate :

$\mathrm{Au}$ cours de l'analyse collective, A. mobilise les mots du modèle des gestes professionnels (GP) : le terme "préoccupation » a deux occurrences; "contrôle » et "tissage " apparaissent une fois; les formules "posture de contrôle" et "posture d'étayage » et « lâcher-prise » une fois également.

Il produit alors la représentation graphique suivante :

Source : Thèse de J.-L. Chauvin dirigée par S. Leblanc \& Y. Soulé.

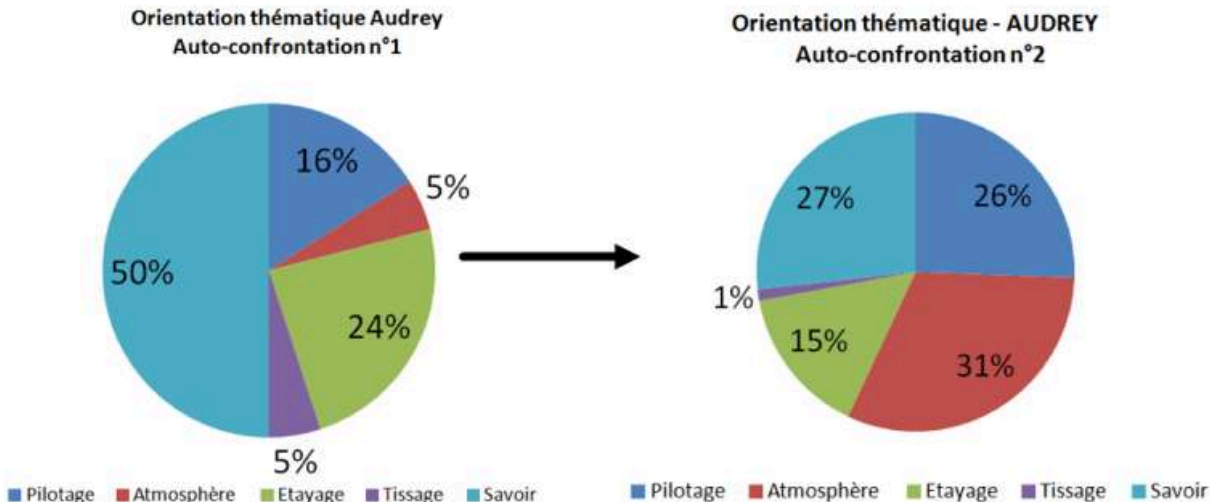

Le travail de recension des gestes montre une évolution significative de la pratique, l'importance accordée aux objets de savoir diminuant au profit d'un rééquilibrage des préoccupations, à l'exception du tissage. Pour l'enseignante débutante comme pour le chercheur, l'analyse par gestes permet d'apprécier le cheminement réflexif.

- Dernier exemple: l'identification des reformulations comme marqueurs d'un savoir-faire professionnel

51 Après un examen théorique systématique des différents types de reformulations, H. Owhadi-Castany (2019), s'appuyant sur le corpus de la recherche Lire et Écrire au CP (Goigoux, 2016), démontre que la fréquence et la distribution de ce phénomène linguistique en fait un "geste professionnel langagier", analyseur de l'activité didactique et témoin de l'expertise enseignante (Chap. XI, p. 343-354). L'extrait cidessous permet de rendre compte d'un autre aspect de l'exploitation du concept de 
geste. Les élèves de $\mathrm{CP}$ réunis en atelier ont décidé d'écrire un élément phrastique proposé par un camarade :

102. Ba. : sinon le loup va te dévorer,+/ [ES]

S'ensuit l'échange suivant :

159. M : L::oup+ [ER]/ qu'est'-ce qu'on écrit,+/ est'-ce que vous pouvez le dire à

Aya.,+/ je crois qu'elle a oublié la phrase, + /

160. Ay. : le loup va te dévorer, + [ER]/

161. Na. : non+ non+n'entre pas+ le loup va te dévorer, + [ER]/

162. Ba. : non+ on l'a fait main(te)nant, + /

163. Li. : $l-+$ ça fait $l-o-u+$ après ça fait $p$ - et après tu fais un point,+/

164. Na. : le loup va te dévorer, $+[\mathrm{ER}] /$ ça va faire ça,+/

165. $\mathrm{M}$ : Alors +

166. Ay. : sinon ça fait loup++[ER]

167. Ba. : loup va te + le loup va te dévorer, $+[\mathrm{ER}] /$

168. $\mathrm{M}$ : on a tous écrit le loup déjà'+ [ER]/

53 En transcrivant au plus près les modalités prosodiques et intonatives ( :» = allongement ; «+» = pause; “" = intonation montante) des énoncés reformulés (ER) à partir de l'énoncé source (ES), on mesure à quel point ce geste langagier "épais » caractérise le travail verbal de l'enseignant encadrant ses élèves dans les premières productions autonomes. Ces dix tours de parole condensent ce qui se joue en termes de postures (de l'enseignant et des élèves), de gestes professionnels de l'enseignant et de gestes d'étude des élèves. Les préoccupations se lisent dans le jeu des reprises. L'étayage initié par l'enseignant pour Aya et le tissage de l'écrit à produire sont partagés par l'ensemble des élèves qui verbalisent les opérations d'encodage, de rappel de la phrase, de suivi des productions de chacun.

\section{Conclusion : un travail à poursuivre}

Les travaux que l'analyse par gestes dans le cadre du multi agenda a initiés ont-ils permis au concept de devenir « le chainon manquant " pour la recherche en didactique du français (Bernié, 2008, p. 237) ? Ils manifestent à l'évidence la volonté d'abandonner " une vision désincarnée de l'activité ». En suscitant une description et une évaluation plus fine des pratiques enseignantes étayées par les apports de la vidéo et des entretiens, le passage par les gestes invite les didacticiens du français à reconsidérer la place des savoirs à l'aune du réel de l'activité, de la régulation des apprentissages, de la gestion des imprévus.

Mais au-delà de cet outillage intellectuel pour parler le métier, l'intérêt du concept offre trois perspectives de développement intéressantes. L'approche par gestes mérite tout d'abord qu'on interroge non seulement le geste comme langage mais comme préalable au langage oral, instituant le langage oral. Évoquant les travaux fondateurs de Jousse (1974) pour qui le geste est à la base d'un « style oral », M. De Certeau reprend une formule de l'anthropologue : «la transmission vivante, gestualisante et oralisante de millénaires générations paysannes » (1970, p. 770). Quand l'enseignant de français s'avance au milieu de la classe, s'arrête, ouvre le livre, porte son regard sur la page écrite, le travail de lecture commence dans sa dimension gestuelle fondatrice d'où procède la parole.

56 Le second point vise à considérer le métier comme un artisanat et par conséquent les gestes qui le définissent - au plan physique, verbal, mental - comme objets et produits 
d'une "conscience matérielle» (Sennett, 2010, p. 166). Cette matérialité a permis à l'équipe du projet TALC ( $\mathrm{Du}$ Texte A La Classe) de montrer que la lecture d'un texte littéraire ne dépend pas d'un dispositif didactique quel qu'il soit, mais d'une configuration dynamique de "textes de lecteurs » - celui de l'enseignant, de chaque élève, de la classe (Louichon, Bazile \& Soulé, 2020).

Le dernier point concerne la dimension éthique du travail d'élucidation du métier : combiner savoir technique et « responsabilité dans l'action »(Bucheton, 2019, p. 31).

\section{BIBLIOGRAPHIE}

ALIN, C. (2010). La Geste Formation. Gestes professionnels et Analyse des pratiques. Paris : Éditions L'Harmattan.

BERNIÉ, J.-P. (2008). « Discussion générale : Le travail sur le geste professionnel : à la recherche du chainon manquant ». In : Bucheton, D. \& Dezutter, O. (dirs), Le développement des gestes professionnels dans l'enseignement du français. Bruxelles : De Boeck, p. 237-245.

BERNIÉ, J.-P. \& GOIGOUX, R. (éds). (2005). « Les gestes professionnels ». La Lettre de la DFLM 36. En ligne : https://www.persee.fr/issue/airdf_1776-7784_2005_num_36_1.

BONNARD, A. \& DETALLE, F. (2014). « Les profils des élèves : typicalisations au service du geste professionnel ». Recherches \& éducations 1, p. 117-128. En ligne : https://doi.org/10.4000/ rechercheseducations.2263.

BRIGAUDIOT, M. (1995). « Apprentissages à l'école maternelle : un aperçu de leur complexité ». Spirale 36, p. 149-156.

BRONCKART, J.-P. \& BULEA BRONCKART, E. (2009). « Le praticien réflexif ou praticien discursif?». Education Canada 49, p. 50-54.

BUCHETON, D., BRONNER, A., BROUSSAL, D., JORRO A. \& LARGUIER, M. (2004). « Les pratiques langagières des enseignants : des savoirs professionnels inédits en formation ». Repères 30, p. 33-53. En ligne : https://doi.org/10.3406/reper.2004.2635.

BUCHETON, D. BRUNET, L. \& LIRIA, A. (2005). « Les gestes professionnels des enseignants : une architecture de gestes complexes ». Actes du $5^{\mathrm{e}}$ Colloque international « Recherche et formation : former des enseignants professionnels, savoirs et compétences ». Nantes.

BUCHETON, D. \& DEZUTTER, O. (dirs). (2008). Le développement des gestes professionnels dans l'enseignement du français. Bruxelles : De Boeck.

BUCHETON, D. (dir.) (2008). L'agir enseignant : des gestes professionnels ajustés. Publication des travaux de l'Équipe de Recherche Technologique en Éducation (ERTE 40). «Conditions et difficultés d'entrée dans les situations d'apprentissage : les langages, vecteurs de la construction des savoirs ", 2004-2006, IUFM de Montpellier. Toulouse : Octarès éditions.

BUCHETON, D. \& SOULÉ, Y. (2009). «Les gestes professionnels et le jeu des postures de l'enseignant dans la classe : un multi-agenda de préoccupations enchâssées ». Éducation \& Didactique 3 (3), p. 29-48. En ligne : https://doi.org/10.4000/educationdidactique.543. 
BUCHETON, D. (2019). Les gestes professionnels dans la classe. Éthique et pratiques pour les temps à venir. Paris : ESF.

BULF, C. (2016). «Étude didactique des gestes professionnels d'un enseignant débutant en formation ». Recherches en didactiques 22, p. 43-64. En ligne $:$ https://doi.org/10.3917/rdid. 022.0043.

BULEA BRONCKART, E. (2010). « Signes, discours et interprétations de l'agir : le rôle des reformulations dans des entretiens portant sur le travail infirmier ». In A. Rabatel (dir.), Les reformulations pluri-sémiotiques en contexte de formation Besançon: Presses universitaires de Franche-Comté, p. 237-253.

BULEA, E. \& BRONCKART, JP. (2010). « Les conditions d'exploitation de l'analyse des pratiques pour la formation des enseignants ». Lingarum Arena 1 (1), p. 43-60.

BULEA BRONCKART, E., RONVEAUX, C. \& VEDRINES, B. (2019). « Pour ne pas conclure : pistes ouvertes pour l'avenir de la discipline ». In : Aeby Daghe, S. et al. (dirs), Didactique du français et construction d'une discipline scientifique. Dialogues avec Bernard Schneuwly. Villeneuve-d'Ascq : Presses du Septentrion, p. 243-252.

BUYSSE, A. \& VANHULLE, S. (2009). «Écriture réflexive et développement professionnel : quels indicateurs? » Questions vives 5 (11), p. 225-245. En ligne : https://doi.org/10.4000/questionsvives. 603.

CÈBE, S. \& GOIGOUX, R. (2009). Lector \& Lectrix : apprendre à comprendre les textes narratifs. CM1, CM2, 6e, Segpa. Paris : Retz.

CHABANNE, J.-C. \& DEZUTTER, O. (dirs). (2011). Les gestes de régulation des apprentissages dans la classe de français : Quelle improvisation professionnelle? Louvain-la-Neuve/Paris : De Boeck Supérieur.

ChABANNE, J.-C., DUPUY, C., DESAULT, M. \& Aigoin, C. (2008). « Les gestes professionnels spécifiques de l'enseignant dans le débat interprétatif : problèmes pour l'analyse et la formation ». Repères 37, $\mathrm{p}$. 227-260. En ligne : https://doi.org/10.4000/reperes.437.

CHEVAlLARD, Y. (2003). « Didactique et formation des enseignants ». Communication aux Journées d'études INRP-GÉDIAPS, Vingt ans de recherche en didactique de l'Éducation Physique et Sportive à l'INRP (1983-2003). Paris, 20 mars.

CIZERON, M. (2010). « Le geste professionnel comme concept et outil d'analyse ». In : M. CIZERON, \& GAL-PETITFAUX, N. (dirs), Analyse des pratiques : expérience et gestes professionnels. Clermont-Ferrand : Presses universitaires Blaise Pascal, p. 257-268.

CLOT, Y. (1999). La fonction psychologique du travail. Paris : Presses universitaires de France.

Clot, Y., \& Fernandez, G. (2005). « Analyse psychologique du mouvement : apport à la compréhension des TMS », Activités 2 (2). En ligne : https://journals.openedition.org/activites/ 1818.

CRAHAY M., \& DUTREVIS M, (2010), Psychologie des apprentissages scolaires. Bruxelles: De Boeck. DE CERTEAU, M. (1990) : L'Invention du quotidien 1. Arts de faire. Paris : Gallimard.

DUFAYS, J.-L. (2005). « La leçon de littérature entre schèmes d'action et gestes professionnels : questions méthodologiques et premiers repérages ». La Lettre de l'AIRDF 36, p. 10-14. En ligne : https://doi.org/10.3406/airdf.2005.1640. 
DUFAYS, J.-L. (2019). « Du geste professionnel au geste didactique ». In : S. Aeby Daghé et al. (dirs), Didactique du français et construction d'une discipline scientifique : Dialogues avec B. Schneuwly. Villeneuve D’Ascq : Presses universitaires du Septentrion, p. 197-210.

DUMEZ, H. (2011). « Qu'est-ce qu'un concept? ». Le Libellio d'Aegis. Libellio d'AEGIS, 7 (1).

DUPUY, C. (2009). Le français tel qu'on l'enseigne. Étude des gestes professionnels de maitres faisant lire un texte de littérature jeunesse au cycle 3. Thèse en sciences du langage et de l'éducation : université de Montpellier.

DUPUY, C., \& GENRE, S. (2020). « Une analyse théorisée de données « authentiques » : enseignement du lexique/vocabulaire en littérature au cycle 3 ». CMLF. En ligne : https://

www.linguistiquefrancaise.org/.

GARCIA-DEBANC, C. (2019). « Le français tel qu'on l'enseigne : objets enseignés en classe de français et modèles disciplinaires ». In : S. Aeby Daghé et al. (dirs), Didactique du français et construction d'une discipline scientifique. Dialogues avec B. Schneuwly ». Villeneuve D'Ascq : Presses universitaires du Septentrion, p. 181-196.

GARCIA-DEBANC, C. \& VOLTEAU, S. (2007). « Formes linguistiques et fonctions des reformulations dans les interactions scolaires ». Recherches linguistiques 29, p. 309-340.

GERRING, J. (1999). « What Makes a Concept Good? A Criterial Framework for Understanding Concept Formation in the Social Sciences ». Polity 31 (3), p. 357-393.

GoIGoux, R. (2005). « Des compétences pour enseigner. Entre objets sociaux et objets de recherche ». In : Talbot, L. \& Bru, M. (dirs), Rennes : Presses universitaires de Rennes, p. 151-168. GoIGOUX, R. (dir.) (2016). Rapport de recherche Lire et Écrire : étude de l'influence des pratiques d'enseignement de la lecture et de l'écriture sur la qualité des premiers apprentissages. Institut Français de l'Éducation, ENS/Université de Lyon. En ligne : http://ife.ens-lyon.fr/ife/recherche/lireecrire.

JAUBERT, M. \& REBIÈRE, M. (2019). « Le scénario langagier didactique, un outil dans le processus de construction des savoirs ? Un exemple : l'enseignement et l'apprentissage de la lecture ». Raisons éducatives 23, p. 153-176. En ligne : https://doi.org/10.3917/raised.023.0153.

JORRO, A. (1998). «L'inscription des gestes professionnels dans l'action ». En Question 19, p. 10-20.

JORRO, A. (2002). Professionnaliser le métier d'enseignant. Paris : ESF.

JORRO, A. (2005). « Le corps parlant de l'enseignant. Entente, malentendus, négociations ». Actes du $9^{e}$ colloque de l'AIRDF, Québec, aout 2004. En ligne : http://www.colloqueairdf.fse.ulaval.ca/ actes/index.php.

JOUSSE, M. (1974). L'anthropologie du geste. Paris : Gallimard.

LHOSTE, Y., CHAMPAGNE, M. \& BULF, C. (2016). « Des gestes professionnels aux gestes langagiers didactiques. Apports d'une recherche comparatiste en didactiques des disciplines pour proposer une nouvelle catégorie d'analyse des pratiques ». Toulouse : Conférence : $4^{\mathrm{e}}$ colloque international de l'ARCD.

LOUICHON, B., BAZILE, S. \& SOULÉ, Y. (2020). « La lecture littéraire professionnelle : une configuration dynamique de textes de lecteurs ». Transpositio 2. En ligne : http://www.transpositio.org/articles/ view/la-lecture-litteraire-professionnelle-une-configuration-dynamique-de-textes-de-lecteurs.

MARLAIR, S. \& DUFAYS, J-L (2008). « Quels gestes dans la classe pour quel enseignementapprentissage de la littérature ? Regard sur quatre leçons de $5^{\mathrm{e}}$ année du secondaire ». In : 
BUCHETON, D. \& DEZUTTER, O. (dirs), Le développement des gestes professionnels dans l'enseignement du français. Toulouse : Octarès éditions, p. 61-82.

NONNON, E. (2017). « Les activités de verbalisation dans la formation des enseignants ». Formation et pratiques d'enseignement en question HS-2, p. 13-31. En ligne : http://revuedeshep.ch/hs-2/.

NONNON, E. (2019). « Notions et questions en mouvement : transpositions, médiations, outils et pratiques langagières ». In : Aeby Daghe et al. (dirs), Didactique du français et construction d'une discipline scientifique : Dialogues avec B. Schneuwly. Villeneuve-d'Asq : Presses universitaires du Septentrion. p. 39-52.

OGDEN, C. \& RICHARDS, I. (1923). The Meaning of Meaning. Londres : Routledge \& Kegan Paul.

OWHADI-CASTANY, H. (2019). Les reformulations orales en contexte d'atelier de production d'écrits au Cours Préparatoire : étude linguistique des jeux de reformulations. Thèse en sciences du langage : Université de Montpellier 3.

PASTRÉ P., MAYEN P. \& VERGNAUd G. (2006). « La didactique professionnelle, note de synthèse ». Revue Française de Pédagogie 154, p. 145-198. En ligne : https://doi.org/10.4000/rfp.157

REUTER, Y. (éd.). (2013)[2007]. Dictionnaire des concepts fondamentaux des didactiques. Bruxelles : De Boeck.

SCHNeuWly, B. (2008). « De l'utilité de la transposition didactique. » In : Chiss, J-L., David J. \& Reuter, Y. (Éds), Didactique du français, fondements d'une discipline. Bruxelles : De Boeck, p. 47-60.

SCHÖN, D. A. (1983). Le praticien réflexif. À la recherche du savoir caché dans l'agir professionnel. Québec : Éditions Logiques.

SENNETT, R. (2010). Ce que sait la main. La culture de l'artisanat. Paris : A. Michel.

SENSEVY, G. (2005). «Sur la notion de geste professionnel ». La Lettre de l'AIRDF 36, p. 4-6. En ligne : https://doi.org/10.3406/airdf.2005.1638.

SENSEVY, G. (2010) « Notes sur la notion de geste d'enseignement ». Travail et formation en éducation 5, p. 1-12. En ligne : http://journals.openedition.org/tfe/1038.

THEUREAU, J. (2004). Le cours d'action : méthode élémentaire. Toulouse : Octarès éditions.

VYGOTSKI, L. (1995)[1934]. Pensée et Langage. Paris : Les Éditions sociales.

WANLIN, P. \& CRAHAY, M. (2012) « La pensée des enseignants pendant l'interaction en classe. Une revue de la littérature anglophone ». Éducation et didactique 1 (6), p. 12-20. En ligne : https:// doi.org/10.4000/educationdidactique.1287.

\section{NOTES}

1. L'équipe de Recherche Technologique pour l'éducation (ERT), sous la direction de D. Bucheton et $\mathrm{A}$. Jorro, réunissait des collègues d'Aix-Marseille, Bordeaux, Montpellier et Toulouse (2002-2008) mais aussi de l'université de Louvain-La-Neuve et de Sherbrooke.

2. Il s'agit de mémoires de master de professeurs stagiaires, d'étudiants en master de Formation de Formateurs, d'étudiants en thèse.

3. La Recherche Lire-écrire CP (Goigoux, 2016). 
4. Thèse en science de l'éducation par J.-L. Chauvin, (dirs. Serge Leblanc, Yves Soulé). Titre provisoire: Analyse de l'activité d'enseignants débutants en contexte de formation hybride.

\section{RÉSUMÉS}

Revenant sur la genèse du modèle des gestes professionnels (Jorro, 1998 ; Bucheton \& Dezuter, 2008) et sur ses échos dans la recherche et la formation (Sensevy, 2010), cette contribution questionne la pertinence d'une notion considérée comme technique professionnelle, mais aussi comme concept et outil d'analyse (Cizeron, 2010). À partir d'extraits de différents corpus relevant de l'enseignement de la lecture, du lire-écrire et de données de la formation, nous montrons comment l'analyse par gestes contribue à la construction des savoirs professionnels didactiques et en particulier des savoirs langagiers. Dès lors, la réflexion ne prétend pas résoudre les tensions relatives à la solidité conceptuelle de la notion de geste mais, s'appuyant sur les nombreuses publications qui l'exploitent dans différents champs disciplinaires (Lhoste, Champagne \& Bulf, 2016), elle tente de faire le point sur son rendement en didactique du français.

Looking back at the genesis of the model of professional gestures (Jorro, 1998; Bucheton \& Dezuter, 2008) and its echoes in research and training (Sensevy, 2010), this contribution questions the relevance of a word as a professional technique, but also as a concept and analytical tool (Cizeron, 2010). From excerpts of different corpus relevant to the teaching of reading, reading-writing and data from training, we show how the analysis by gestures contributes to the construction of didactic professional knowledge and in particular language knowledge. Consequently, the reflection does not intend to resolve the tensions relating to the conceptual solidity of the notion of gesture but, relying on the numerous publications that exploit it in different disciplinary fields (Lhoste, Champagne \& Bulf, 2016), it tries to take stock of its performance in teaching French.

\section{INDEX}

Mots-clés : concepts, gestes professionnels, agir enseignant, outils d'analyse, modèle didactique, recherche-formation

Keywords : concepts, professional gestures, teacher action, didactic model, analysis tools, research-training

\section{AUTEURS}

\section{CATHERINE DUPUY}

LIRDEF, Université Montpellier, Université Paul-Valéry Montpellier 3, Montpellier, Faculté d'Éducation, France 


\section{YVES SOULÉ}

LIRDEF, Université Montpellier, Université Paul-Valéry Montpellier 3, Montpellier, Faculté d'Éducation, France 